\title{
Weighted Simpson type inequalities for h-convex functions
}

\section{Marian Matłoka}

Poznań University of Economics and Business, aleja Niepodległości 10, Poznań, Poland.

Communicated by J. Pecaric

\begin{abstract}
In this paper we establish some weighted Simpson type inequalities for functions whose derivatives in absolute value are h-convex. (C) 2017 All rights reserved.
\end{abstract}

Keywords: Simpson inequality, weighted inequalities, h-convex function.

2010 MSC: 26D15, 26D10, 41A55.

\section{Introduction}

The Simpson inequality states that if $f$ exists and is bounded on $(a, b)$, then

$$
\left|\int_{a}^{b} f(x) d x-\frac{b-a}{3}\left[\frac{f(a)+f(b)}{2}+2 f\left(\frac{a+b}{2}\right)\right]\right| \leqslant \frac{1}{2880}\left\|f^{(4)}\right\|_{\infty} \cdot(b-a)^{4},
$$

where

$$
\left\|f^{(4)}\right\|_{\infty}:=\sup _{t \in(a, b)}\left|f^{(4)}(t)\right|<\infty .
$$

In [3], Dragomir et al. proved the following inequality.

Theorem 1.1. Suppose $\mathrm{f}:[\mathrm{a}, \mathrm{b}] \rightarrow \mathrm{R}$ is a differentiable mapping whose derivative is continuous on $(\mathrm{a}, \mathrm{b})$ and $f^{\prime} \in \mathrm{L}([\mathrm{a}, \mathrm{b}])$. Then the following inequality holds:

$$
\left|\frac{1}{3}\left[\frac{f(a)+f(b)}{2}+2 f\left(\frac{a+b}{2}\right)\right]-\frac{1}{b-a} \int_{a}^{b} f(x) d x\right| \leqslant \frac{b-a}{3}\left\|f^{\prime}\right\|_{1}
$$

where

$$
\left\|f^{\prime}\right\|_{1}=\int_{a}^{b}\left|f^{\prime}(x)\right| d x
$$

Email address: marian.matloka@ue.poznan.pl (Marian Matłoka)

doi:10.22436/jnsa.010.11.15 
In [9], Sarikaya et al. obtained inequalities for differentiable convex mappings. The main inequality is as follows.

Theorem 1.2. Let $\mathrm{f}: \mathrm{I} \subset \mathrm{R} \rightarrow \mathrm{R}$ be a differentiable mapping on $\mathrm{I}^{0}$ such that $\mathrm{f}^{\prime} \in \mathrm{L}([\mathrm{a}, \mathrm{b}])$, where $\mathrm{a}, \mathrm{b} \in \mathrm{I}^{0}$ with $\mathrm{a}<\mathrm{b}$. If $\left|\mathrm{f}^{\prime}\right|^{\mathrm{q}}$ is convex on $[\mathrm{a}, \mathrm{b}], \mathrm{q}>1$, then the following inequality holds:

$$
\begin{aligned}
& \left|\frac{1}{6}\left[f(a)+4 f\left(\frac{a+b}{2}\right)+f(b)\right]-\frac{1}{b-a} \int_{a}^{b} f(x) d x\right| \\
& \quad \leqslant \frac{b-a}{12}\left(\frac{1+2^{p+1}}{3(p+1)}\right)^{\frac{1}{p}}\left\{\left(\frac{3\left|f^{\prime}(b)\right|^{q}+\left|f^{\prime}(a)\right|^{q}}{4}\right)^{\frac{1}{q}}+\left(\frac{\left|f^{\prime}(b)\right|^{q}+3\left|f^{\prime}(a)\right|^{q}}{4}\right)^{\frac{1}{q}}\right\},
\end{aligned}
$$

where

$$
\frac{1}{p}+\frac{1}{q}=1
$$

In [10], Sarikaya et al. obtained the following inequality for s-convex functions.

Theorem 1.3. Let $\mathrm{f}: \mathrm{I} \subset[0, \infty) \rightarrow \mathrm{R}$ be a differentiable mapping on $\mathrm{I}^{0}$ such that $\mathrm{f}^{\prime} \in \mathrm{L}([\mathrm{a}, \mathrm{b}])$, where $\mathrm{a}, \mathrm{b} \in \mathrm{I}^{0}$ with $\mathrm{a}<\mathrm{b}$. If $\left|\mathrm{f}^{\prime}\right|^{\mathrm{q}}$ is s-convex on $[\mathrm{a}, \mathrm{b}]$, for some fixed $\mathrm{s} \in(0,1]$ and $\mathrm{q}<1$, then the following inequality holds:

$$
\begin{aligned}
& \left|\frac{1}{6}\left[f(a)+4 f\left(\frac{a+b}{2}\right)+f(b)\right]-\frac{1}{b-a} \int_{a}^{b} f(x) d x\right| \\
& \quad \leqslant \frac{b-a}{12}\left(\frac{1+2^{p+1}}{3(p+1)}\right)^{\frac{1}{p}}\left\{\left(\frac{\left|f^{\prime}(b)\right|^{q}+\left|f^{\prime}\left(\frac{a+b}{2}\right)\right|^{q}}{4}\right)^{\frac{1}{q}}+\left(\frac{\left|f^{\prime}(a)\right|^{q}+\left|f^{\prime}\left(\frac{a+b}{2}\right)\right|^{q}}{4}\right)^{\frac{1}{q}}\right\},
\end{aligned}
$$

where $\frac{1}{p}+\frac{1}{q}=1$.

For recent refinements, counterparts, generalizations, and inequalities of Simpson type, see $[1-7,9,10]$ and $[11,12]$.

In 2007, Varošanec in [13] introduced a large class of functions, the so-called h-convex functions. This class contains several well-known classes of functions such as non-negative convex functions, s-convex in the second sense, Godunova Levin functions and P-functions. This class is defined in the following way: a function $f: I \rightarrow R, \emptyset \neq I \subset R$ being an interval is called h-convex, if

$$
f(t x+(1-t) y) \leqslant h(t) f(x)+h(1-t) f(y),
$$

holds for all $x, y \in I, t \in(0,1)$, where $h: J \rightarrow R, h \neq 0$ and $J$ is an interval, $(0,1) \subseteq J$.

In [8], Sarikaya et al. proved that for h-convex function the following variant of the Hadamard inequality is fulfilled:

$$
\frac{1}{2 h\left(\frac{1}{2}\right)} f\left(\frac{a+b}{2}\right) \leqslant \frac{1}{b-a} \int_{a}^{b} f(x) d x \leqslant[f(a)+f(b)] \cdot \int_{0}^{1} h(t) d t .
$$

The main purpose of the present paper is to establish new weighted Simpson type inequalities for functions whose derivatives in absolute value are $h$-convex.

\section{Main result}

In order to prove our main theorems, we need the following lemma. 
Lemma 2.1. Let $\mathrm{f}:[\mathrm{a}, \mathrm{b}] \rightarrow \mathrm{R}$ be a differentiable mapping on $(\mathrm{a}, \mathrm{b})$ with $\mathrm{a}<\mathrm{b}$ and let $\mathrm{w}:[\mathrm{a}, \mathrm{b}] \rightarrow \mathrm{R}$ be symmetric mapping to $\frac{\mathrm{a}+\mathrm{b}}{2}$. If $\mathrm{f}^{\prime}, w \in \mathrm{L}([\mathrm{a}, \mathrm{b}])$, then the following indentity holds:

$$
\begin{aligned}
& \frac{1}{6(b-a)}\left[f(a)+f(b)+4 f\left(\frac{a+b}{2}\right)\right] \int_{a}^{b} w(x) d x-\frac{1}{b-a} \int_{a}^{b} w(x) f(x) d x \\
& =\frac{b-a}{2}\left\{\int_{0}^{1}\left[\frac{1}{2} \int_{0}^{t} w\left(\frac{1-s}{2} a+\frac{1+s}{2} b\right) d s-\frac{1}{3} \int_{0}^{1} w\left(\frac{1-s}{2} a+\frac{1+s}{2} b\right) d s\right] f^{\prime}\left(\frac{1-t}{2} a+\frac{1+t}{2} b\right) d t\right. \\
& \left.+\int_{0}^{1}\left[\frac{1}{3} \int_{0}^{1} w\left(\frac{1+s}{2} a+\frac{1-s}{2} b\right) d s-\frac{1}{2} \int_{0}^{t} w\left(\frac{1+s}{2} a+\frac{1-s}{2} b\right) d s\right] f^{\prime}\left(\frac{1+t}{2} a+\frac{1-t}{2} b\right) d t\right\} .
\end{aligned}
$$

Proof. By integration by parts and changing the variables, we get

$$
\begin{aligned}
I_{1}= & \int_{0}^{1}\left[\frac{1}{2} \int_{0}^{t} w\left(\frac{1-s}{2} a+\frac{1+s}{2} b\right) d s-\frac{1}{3} \int_{0}^{1} w\left(\frac{1-s}{2} a+\frac{1+s}{2} b\right) d s\right] f^{\prime}\left(\frac{1-t}{2} a+\frac{1+t}{2} b\right) d t \\
= & \left.\frac{2}{b-a}\left[\frac{1}{2} \int_{0}^{t} w\left(\frac{1-s}{2} a+\frac{1+s}{2} b\right) d s-\frac{1}{3} \int_{0}^{1} w\left(\frac{1-s}{2} a+\frac{1+s}{2} b\right) d s\right] f\left(\frac{1-t}{2} a+\frac{1+t}{2} b\right)\right|_{0} ^{1} \\
& -\frac{1}{b-a} \int_{0}^{1} w\left(\frac{1-t}{2} a+\frac{1+t}{2} b\right) f\left(\frac{1-t}{2} a+\frac{1+t}{2} b\right) d t \\
= & \frac{2}{b-a}\left[\frac{1}{6} f(b)+\frac{1}{3} f\left(\frac{a+b}{2}\right)\right] \int_{0}^{1} w\left(\frac{1-s}{2} a+\frac{1+s}{2} b\right) d s \\
& -\frac{1}{b-a} \int_{0}^{1} w\left(\frac{1-t}{2} a+\frac{1+t}{2} b\right) f\left(\frac{1-t}{2} a+\frac{1+t}{2} b\right) d t \\
= & \frac{4}{(b-a)^{2}}\left[\frac{1}{6} f(b)+\frac{1}{3} f\left(\frac{a+b}{2}\right)\right] \int_{\frac{a+b}{2}}^{b} w(x) d x-\frac{2}{(b-a)^{2}} \int_{\frac{a+b}{2}}^{b} w(x) f(x) d x .
\end{aligned}
$$

and similarly

$$
\begin{aligned}
\mathrm{I}_{2}= & \int_{0}^{1}\left[\frac{1}{3} \int_{0}^{1} w\left(\frac{1+\mathrm{s}}{2} \mathrm{a}+\frac{1-\mathrm{s}}{2} \mathrm{~b}\right) \mathrm{d} s-\frac{1}{2} \int_{0}^{\mathrm{t}} w\left(\frac{1+\mathrm{s}}{2} \mathrm{a}+\frac{1-\mathrm{s}}{2} \mathrm{~b}\right) \mathrm{ds}\right] \mathrm{f}^{\prime}\left(\frac{1+\mathrm{t}}{2} \mathrm{a}+\frac{1-\mathrm{t}}{2} \mathrm{~b}\right) \mathrm{dt} \\
= & -\left.\frac{2}{\mathrm{~b}-\mathrm{a}}\left[\frac{1}{3} \int_{0}^{1} w\left(\frac{1+\mathrm{s}}{2} \mathrm{a}+\frac{1-\mathrm{s}}{2} \mathrm{~b}\right) \mathrm{ds}-\frac{1}{2} \int_{0}^{\mathrm{t}} w\left(\frac{1+\mathrm{s}}{2} \mathrm{a}+\frac{1-\mathrm{s}}{2} \mathrm{~b}\right) \mathrm{ds}\right] \mathrm{f}\left(\frac{1+\mathrm{t}}{2} \mathrm{a}+\frac{1-\mathrm{t}}{2} \mathrm{~b}\right)\right|_{0} ^{1} \\
& -\frac{1}{\mathrm{~b}-\mathrm{a}} \int_{0}^{1} w\left(\frac{1+\mathrm{t}}{2} \mathrm{a}+\frac{1-\mathrm{t}}{2} \mathrm{~b}\right) \mathrm{f}\left(\frac{1+\mathrm{t}}{2} \mathrm{a}+\frac{1-\mathrm{t}}{2} \mathrm{~b}\right) \mathrm{dt} \\
= & -\frac{2}{\mathrm{~b}-\mathrm{a}}\left[-\frac{1}{6} \mathrm{f}(\mathrm{a})-\frac{1}{3} \mathrm{f}\left(\frac{\mathrm{a}+\mathrm{b}}{2}\right)\right] \int_{0}^{1} w\left(\frac{1+s}{2} \mathrm{a}+\frac{1-\mathrm{s}}{2} \mathrm{~b}\right) \mathrm{ds}
\end{aligned}
$$




$$
\begin{aligned}
& -\frac{1}{b-a} \int_{0}^{1} w\left(\frac{1+t}{2} a+\frac{1-t}{2} b\right) f\left(\frac{1+t}{2} a+\frac{1-t}{2} b\right) d t \\
= & \frac{4}{(b-a)^{2}}\left[\frac{1}{6} f(a)+\frac{1}{3} f\left(\frac{a+b}{2}\right)\right] \int_{a}^{\frac{a+b}{2}} w(x) d x-\frac{2}{(b-a)^{2}} \int_{a}^{\frac{a+b}{2}} w(x) f(x) d x .
\end{aligned}
$$

Since $w(x)$ is symmetric to $\frac{a+b}{2}$, we have

$$
\int_{a}^{\frac{a+b}{2}} w(x) d x=\int_{\frac{a+b}{2}}^{b} w(x) d x=\frac{1}{2} \int_{a}^{b} w(x) d x
$$

Thus, we can write

$$
\frac{b-a}{2}\left(I_{1}+I_{2}\right)=\frac{1}{6(b-a)}\left[f(a)+f(b)+4 f\left(\frac{a+b}{2}\right)\right] \int_{a}^{b} w(x) d x+\frac{1}{b-a} \int_{a}^{b} w(x) f(x) d x
$$

which completes the proof.

Throughout this paper, let $\|w\|_{[a, b], \infty}=\sup _{x \in[a, b]}|w(x)|$, for the continuous function $w:[a, b] \rightarrow R$. Now, we are ready to state and prove our results.

Theorem 2.2. Let $\mathrm{f}:[\mathrm{a}, \mathrm{b}] \rightarrow \mathrm{R}$ be a differentiable mapping on $(\mathrm{a}, \mathrm{b})$ and $\mathrm{f}^{\prime} \in \mathrm{L}([\mathrm{a}, \mathrm{b}])$ with $\mathrm{a}<\mathrm{b}$ and $w:[\mathrm{a}, \mathrm{b}] \rightarrow \mathrm{R}$ be continuous and symmetric to $\frac{\mathrm{a}+\mathrm{b}}{2}$. If $\left|\mathrm{f}^{\prime}\right|$ is $\mathrm{h}$-convex on $[\mathrm{a}, \mathrm{b}]$, then the following inequality holds:

$$
\begin{gathered}
\left|\frac{1}{6(b-a)}\left[f(a)+f(b)+4 f\left(\frac{a+b}{2}\right)\right] \int_{a}^{b} w(x) d x-\frac{1}{b-a} \int_{a}^{b} w(x) f(x) d x\right| \\
\leqslant(b-a)\|w\|_{[a, b], \infty}\left[\left|f^{\prime}(a)\right|+\left|f^{\prime}(b)\right|\right] \int_{0}^{1} h(t) d t .
\end{gathered}
$$

Proof. From Lemma 2.1 and since $\left|f^{\prime}\right|$ is $h$-convex on $[a, b]$ we have

$$
\begin{aligned}
& \left|\frac{1}{6(b-a)}\left[f(a)+f(b)+4 f\left(\frac{a+b}{2}\right)\right] \int_{a}^{b} w(x) d x-\frac{1}{b-a} \int_{a}^{b} w(x) f(x) d x\right| \\
& \leqslant \frac{b-a}{2}\left\{\int_{0}^{1}\left|\frac{1}{2} \int_{0}^{t} w\left(\frac{1-s}{2} a+\frac{1+s}{2} b\right) d s-\frac{1}{3} \int_{0}^{1} w\left(\frac{1-s}{2} a+\frac{1+s}{2} b\right) d s\right| \cdot\left|f^{\prime}\left(\frac{1-t}{2} a+\frac{1+t}{2} b\right)\right| d t\right. \\
& \left.\quad+\int_{0}^{1}\left|\frac{1}{3} \int_{0}^{1} w\left(\frac{1+s}{2} a+\frac{1-s}{2} b\right) d s-\frac{1}{2} \int_{0}^{1} w\left(\frac{1+s}{2} a+\frac{1-s}{2} b\right) d s\right| \cdot\left|f^{\prime}\left(\frac{1+t}{2} a+\frac{1-t}{2} b\right)\right| d t\right\} \\
& \leqslant \frac{b-a}{2}\|w\|_{[a, b], \infty}\left\{\int_{0}^{1}\left|\frac{1}{2} \int_{0}^{t} d s-\frac{1}{3} \int_{0}^{1} d s\right| \cdot\left|f^{\prime}\left(\frac{1-t}{2} a+\frac{1+t}{2} b\right)\right| d t\right.
\end{aligned}
$$




$$
\begin{aligned}
& \left.+\int_{0}^{1}\left|\frac{1}{3} \int_{0}^{1} d s-\frac{1}{2} \int_{0}^{t} d s\right| \cdot\left|f^{\prime}\left(\frac{1+t}{2} a+\frac{1-t}{2} b\right)\right| d t\right\} \\
& \leqslant \frac{b-a}{2}\|w\|_{[a, b], \infty}\left\{\int_{0}^{1}\left(h\left(\frac{1-t}{2}\right)\left|f^{\prime}(a)\right|+h\left(\frac{1+t}{2}\right)\left|f^{\prime}(b)\right|\right) d t\right. \\
& \left.+\int_{0}^{1}\left(h\left(\frac{1+t}{2}\right)\left|f^{\prime}(a)\right|+h\left(\frac{1-t}{2}\right)\left|f^{\prime}(b)\right|\right) d t\right\} \\
& =(b-a)\|w\|_{[a, b], \infty}\left[\left|f^{\prime}(a)\right|+\left|f^{\prime}(b)\right|\right] \int_{0}^{1} h(t) d t
\end{aligned}
$$

where

$$
\|w\|_{\left[\mathrm{a}, \frac{\mathrm{a}+\mathrm{b}}{2}\right], \infty}=\|w\|_{\left[\frac{\mathrm{b}+\mathrm{a}}{2}, \mathrm{~b}\right], \infty}=\|w\|_{[\mathrm{a}, \mathrm{b}], \infty}
$$

and

$$
\left|\frac{1}{2} \int_{0}^{\mathrm{t}} \mathrm{d} s-\frac{1}{3} \int_{0}^{1} \mathrm{~d} s\right|=\left|\frac{1}{3} \int_{0}^{1} \mathrm{~d} s-\frac{1}{2} \int_{0}^{\mathrm{t}} \mathrm{d} s\right| \leqslant \frac{1}{3}
$$

for each $t \in[0,1]$. This completes the proof.

Corollary 2.3. In Theorem 2.2, if we take $\mathrm{h}(\mathrm{t})=\mathrm{t}$, then inequality (2.1) becomes the following inequality for convex functions:

$$
\begin{aligned}
& \mid \frac{1}{6(b-a)} {\left[f(a)+f(b)+4 f\left(\frac{a+b}{2}\right)\right] \int_{a}^{b} w(x) d x-\frac{1}{b-a} \int_{a}^{b} w(x) f(x) d x \mid } \\
& \leqslant \frac{b-a}{2}\|w\|_{[a, b], \infty}\left[\left|f^{\prime}(a)\right|+\left|f^{\prime}(b)\right|\right] .
\end{aligned}
$$

Corollary 2.4. Suppose $\mathrm{h}(\mathrm{t})=\mathrm{t}^{\mathrm{s}}, \mathrm{s} \in(0,1]$ in Theorem 2.2, we have the following inequality for s-convex functions:

$$
\begin{gathered}
\left|\frac{1}{6(b-a)}\left[f(a)+f(b)+4 f\left(\frac{a+b}{2}\right)\right] \int_{a}^{b} w(x) d x-\frac{1}{b-a} \int_{a}^{b} w(x) f(x) d x\right| \\
\leqslant \frac{b-a}{(s+1)}\|w\|_{[a, b], \infty}\left[\left|f^{\prime}(a)\right|+\left|f^{\prime}(b)\right|\right] .
\end{gathered}
$$

Remark 2.5. If we set $h(t)=t$ in the proof of Theorem 2.2, then using the fact that

$$
\int_{0}^{1}\left|\frac{1}{2} \int_{0}^{t} d s-\frac{1}{3} \int_{0}^{1} d s\right| d t=\int_{0}^{1}\left|\frac{1}{3} \int_{0}^{1} d s-\frac{1}{2} \int_{0}^{t} d s\right| d t=\int_{0}^{1}\left|\frac{1}{2} t-\frac{1}{3}\right| d t=\int_{0}^{1}\left|\frac{1}{3}-\frac{1}{2} t\right| d t=\frac{5}{36},
$$

we obtain the following inequality:

$$
\begin{gathered}
\left|\frac{1}{6(b-a)}\left[f(a)+f(b)+4 f\left(\frac{a+b}{2}\right)\right] \int_{a}^{b} w(x) d x-\frac{1}{b-a} \int_{a}^{b} w(x) f(x) d x\right| \\
\leqslant \frac{5}{72}\|w\|_{[a, b], \infty}\left[\left|f^{\prime}(a)\right|+\left|f^{\prime}(b)\right|\right] .
\end{gathered}
$$


Theorem 2.6. Let $\mathrm{f}:[\mathrm{a}, \mathrm{b}] \rightarrow \mathrm{R}$ be a differentiable mapping on $(\mathrm{a}, \mathrm{b})$ and $\mathrm{f}^{\prime} \in \mathrm{L}([\mathrm{a}, \mathrm{b}])$ with $\mathrm{a}<\mathrm{b}$ and $w:[\mathrm{a}, \mathrm{b}] \rightarrow \mathrm{R}$ be continuous and symmetric to $\frac{\mathrm{a}+\mathrm{b}}{2}$. If $\left|\mathrm{f}^{\prime}\right|^{\mathrm{q}}$ is $\mathrm{h}$-convex on $[\mathrm{a}, \mathrm{b}]$ and $\mathrm{q}>1$, then the following inequality holds:

$$
\begin{aligned}
& \left|\frac{1}{6(b-a)}\left[f(a)+f(b)+4 f\left(\frac{a+b}{2}\right)\right] \int_{a}^{b} w(x) d x-\frac{1}{b-a} \int_{a}^{b} w(x) f(x) d x\right| \\
& \leqslant \frac{b-a}{12}\|w\|_{[a, b], \infty} \cdot\left(\frac{1+2^{p+1}}{3(p+1)}\right)^{\frac{1}{p}} \cdot 2^{\frac{1}{q}}\left\{\left(\left|f^{\prime}(a)\right|^{q} \int_{0}^{\frac{1}{2}} h(t) d t\right.\right. \\
& \left.\left.+\left|f^{\prime}(b)\right|^{q} \int_{\frac{1}{2}}^{1} h(t) d t\right)^{\frac{1}{q}}+\left(\left|f^{\prime}(a)\right|^{q} \int_{\frac{1}{2}}^{1} h(t) d t+\left|f^{\prime}(b)\right|^{q} \int_{0}^{\frac{1}{2}} h(t) d t\right)^{\frac{1}{q}}\right\},
\end{aligned}
$$

where $\frac{1}{\mathrm{p}}+\frac{1}{\mathrm{q}}=1$.

Proof. From Lemma 2.1 and using the Hölder's integrals inequality and the h-convexity of $\left|f^{\prime}\right| q$, we have

$$
\begin{aligned}
& \left|\frac{1}{6(b-a)}\left[f(a)+f(b)+4 f\left(\frac{a+b}{2}\right)\right] \int_{a}^{b} w(x) d x-\frac{1}{b-a} \int_{a}^{b} w(x) f(x) d x\right| \\
& \leqslant \frac{(b-a)}{2}\left\{\left(\int_{0}^{1}\left|\frac{1}{2} \int_{0}^{t} w\left(\frac{1-s}{2} a+\frac{1+s}{2} b\right) d s-\frac{1}{3} \int_{0}^{1} w\left(\frac{1-s}{2} a+\frac{1+s}{2} b\right) d s\right|^{p} d t\right)^{\frac{1}{p}}\right. \\
& \quad \times\left(\int_{0}^{1}\left|f^{\prime}\left(\frac{1-t}{2} a+\frac{1+t}{2} b\right)\right|^{q} d t\right)^{\frac{1}{q}} \\
& \quad+\left(\int_{0}^{1}\left|\frac{1}{3} \int_{0}^{1} w\left(\frac{1+s}{2} a+\frac{1-s}{2} b\right) d s-\frac{1}{2} \int_{0}^{t} w\left(\frac{1+s}{2} a+\frac{1-s}{2} b\right) d s\right|^{p} d t\right)^{\frac{1}{p}} \\
& \left.\quad \times\left(\int_{0}^{1}\left|f^{\prime}\left(\frac{1+t}{2} a+\frac{1-t}{2} b\right)\right|^{q} d t\right)^{\frac{1}{q}}\right\} \\
& \leqslant
\end{aligned}
$$




$$
\left.+\left(\left|f^{\prime}(a)\right|^{q} \int_{\frac{1}{2}}^{1} h(t) d t+\left|f^{\prime}(b)\right|^{q} \int_{0}^{\frac{1}{2}} h(t) d t\right)\right\},
$$

where

$$
\int_{0}^{1}\left|\frac{1}{2} \mathrm{t}-\frac{1}{3}\right|^{\mathrm{p}} \mathrm{dt}=\int_{0}^{1}\left|\frac{1}{3}-\frac{1}{2} \mathrm{t}\right|^{\mathrm{p}} \mathrm{dt}=\frac{2+2^{p+2}}{(\mathrm{p}+1) \cdot 6^{\mathrm{p}+1}}
$$

which completes the proof.

Corollary 2.7. If we set $\mathrm{h}(\mathrm{t})=\mathrm{t}$ in Theorem 2.6, we obtain the inequality for convex functions:

$$
\begin{aligned}
& \left|\frac{1}{6(b-a)}\left[f(a)+f(b)+4 f\left(\frac{a+b}{2}\right)\right] \int_{a}^{b} w(x) d x-\frac{1}{b-a} \int_{a}^{b} w(x) f(x) d x\right| \\
& \leqslant \frac{b-a}{12}\|w\|_{[a, b], \infty}\left(\frac{1+2^{p+1}}{3(p+1)}\right)\left(\frac{1}{4}\right)^{\frac{1}{q}}\left\{\left(\left|f^{\prime}(a)\right|^{q}+3\left|f^{\prime}(b)\right|^{q}\right)^{\frac{1}{q}}+\left(3\left|f^{\prime}(a)\right|^{q}+\left|f^{\prime}(b)\right|^{q}\right)^{\frac{1}{q}}\right\} .
\end{aligned}
$$

Corollary 2.8. If we set $\mathrm{h}(\mathrm{t})=\mathrm{t}, \mathrm{s} \in(0,1]$ in Theorem 2.6 , we obtain the inequality for s-convex functions:

$$
\begin{aligned}
& \left|\frac{1}{6(b-a)}\left[f(a)+f(b)+4 f\left(\frac{a+b}{2}\right)\right] \int_{a}^{b} w(x) d x-\frac{1}{b-a} \int_{a}^{b} w(x) f(x) d x\right| \\
& \leqslant \frac{b-a}{12}\|w\|_{[a, b], \infty}\left(\frac{1+2^{p+1}}{3(p+1)}\right)\left(\frac{1}{4}\right)^{\frac{1}{q}} \cdot\left(\frac{2}{s+1}\right)^{\frac{1}{q}}\left\{\left(\left|f^{\prime}(a)\right|^{q}\left(\frac{1}{2}\right)^{s+1}+\left|f^{\prime}(b)\right|^{q}\left(1-\left(\frac{1}{2}\right)^{s+1}\right)\right)^{\frac{1}{q}}\right. \\
& \left.+\left(\left|f^{\prime}(a)\right|^{q}\left(1-\left(\frac{1}{2}\right)^{s+1}\right)+\left|f^{\prime}(b)\right|^{q}\left(\frac{1}{2}\right)^{s+1}\right)^{\frac{1}{q}}\right\} .
\end{aligned}
$$

Remark 2.9. If we set $h(t)=t$ and $w(x)=1$ for each $x \in[a, b]$ in Theorem 2.6, then inequality (2.2) reduces to the inequality (1.1).

Remark 2.10. If we set $h(t)=t^{s}, s \in(0,1]$ and $w(x)=1$ for each $x \in[a, b]$ in Theorem 2.6, then inequality (2.2) reduces to the inequality [10, Eq. (2.9)].

Theorem 2.11. Let $\mathrm{f}:[\mathrm{a}, \mathrm{b}] \rightarrow \mathrm{R}$ be a differentiable mapping on $(\mathrm{a}, \mathrm{b})$ and $\mathrm{f}^{\prime} \in \mathrm{L}([\mathrm{a}, \mathrm{b}])$ with $\mathrm{a}<\mathrm{b}$ and $w:[a, b] \rightarrow R$ be continuous and symmetric to $\frac{\mathrm{a}+\mathrm{b}}{2}$. If $\left|\mathrm{f}^{\prime}\right|^{\mathrm{q}}$ is $\mathrm{h}$-convex on $[\mathrm{a}, \mathrm{b}]$ and $\mathrm{q} \geqslant 1$, then the following inequality holds:

$$
\begin{aligned}
& \left|\frac{1}{6(b-a)}\left[f(a)+f(b)+4 f\left(\frac{a+b}{2}\right)\right] \int_{a}^{b} w(x) d x-\frac{1}{b-a} \int_{a}^{b} w(x) f(x) d x\right| \\
& \leqslant \frac{b-a}{12}\|w\|_{[a, b], \infty}\left(\frac{5}{36}\right)^{1-\frac{1}{q}} \cdot\left(\frac{2}{3}\right)^{\frac{1}{q}}\left\{\left(\left|f^{\prime}(a)\right|^{q} \int_{0}^{\frac{1}{2}} h(t) d t+\left|f^{\prime}(b)\right| q \int_{\frac{1}{2}}^{1} h(t) d t\right)^{\frac{1}{q}}\right. \\
& \left.+\left(\left|f^{\prime}(a)\right|^{q} \int_{\frac{1}{2}}^{1} h(t) d t+\left|f^{\prime}(b)\right|^{q} \int_{0}^{\frac{1}{2}} h(t) d t\right)^{\frac{1}{q}}\right\} .
\end{aligned}
$$


Proof. From Lemma 2.1 and the power mean inequality, we have that the following inequality holds:

$$
\begin{aligned}
& \left|\frac{1}{6(b-a)}\left[f(a)+f(b)+4 f\left(\frac{a+b}{2}\right)\right] \int_{a}^{b} w(x) d x-\frac{1}{b-a} \int_{a}^{b} w(x) f(x) d x\right| \\
& \leqslant \frac{b-a}{2}\left\{\left(\int_{0}^{1}\left|\frac{1}{2} \int_{0}^{t} w\left(\frac{1-s}{2} a+\frac{1+s}{2} b\right) d s-\frac{1}{3} \int_{0}^{1} w\left(\frac{1-s}{2} a+\frac{1+s}{2} b\right) d s\right| d t\right)^{\frac{1}{1-q}}\right. \\
& \times\left(\int_{0}^{1}\left|\frac{1}{2} \int_{0}^{t} w\left(\frac{1-s}{2} a+\frac{1+s}{2} b\right) d s-\frac{1}{3} \int_{0}^{1} w\left(\frac{1-s}{2} a+\frac{1+s}{2} b\right) d s \| f^{\prime}\left(\frac{1-t}{2} a+\frac{1+t}{2} b\right)\right|^{q} d t\right)^{\frac{1}{q}} \\
& +\left(\int_{0}^{1}\left|\frac{1}{3} \int_{0}^{1} w\left(\frac{1+s}{2} a+\frac{1-s}{2} b\right) d s-\frac{1}{2} \int_{0}^{t} w\left(\frac{1+s}{2} a+\frac{1-s}{2} b\right) d s\right| d t\right)^{1-\frac{1}{q}} \\
& \left.\times\left(\int_{0}^{1}\left|\frac{1}{3} \int_{0}^{1} w\left(\frac{1+s}{2} a+\frac{1-s}{2} b\right) d s-\frac{1}{2} \int_{0}^{t} w\left(\frac{1+s}{2} a+\frac{1-s}{2} b\right) d s \| f^{\prime}\left(\frac{1+t}{2} a+\frac{1+t}{2} b\right)\right|^{q} d t\right)^{\frac{1}{q}}\right\} .
\end{aligned}
$$

By the h-convexity of $\left|f^{\prime}\right|^{q}$ and using the facts that

$$
\left|\frac{1}{2} \int_{0}^{\mathrm{t}} \mathrm{ds}-\frac{1}{3} \int_{0}^{1} \mathrm{ds}\right|=\left|\frac{1}{3} \int_{0}^{1} \mathrm{~d} \mathrm{~s}-\frac{1}{2} \int_{0}^{\mathrm{t}} \mathrm{ds}\right|=\left|\frac{1}{2} \mathrm{t}-\frac{1}{3}\right| \leqslant \frac{1}{3}
$$

for all $t \in[0,1]$ we have

$$
\begin{aligned}
& \int_{0}^{1}\left|\frac{1}{2} \int_{0}^{t} w\left(\frac{1-s}{2} a+\frac{1+s}{2} b\right) d s-\frac{1}{3} \int_{0}^{1} w\left(\frac{1-s}{2} a+\frac{1+s}{2} b\right) d s\right| \cdot\left|f^{\prime}\left(\frac{1-t}{2} a+\frac{1+t}{2} b\right)\right|^{q} d t \\
& \leqslant\|w\|_{[a, b], \infty} \frac{1}{3}\left(\left|f^{\prime}(a)\right|^{q} \int_{0}^{1} h\left(\frac{1-t}{2}\right) d t+\left|f^{\prime}(b)\right|^{q} \int_{0}^{1} h\left(\frac{1+t}{2}\right) d t\right),
\end{aligned}
$$

and

$$
\begin{aligned}
& \int_{0}^{1}\left|\frac{1}{3} \int_{0}^{1} w\left(\frac{1+s}{2} a+\frac{1-s}{2} b\right) d s-\frac{1}{2} \int_{0}^{t} w\left(\frac{1+s}{2} a+\frac{1-s}{2} b\right) d s\right| \cdot\left|f^{\prime}\left(\frac{1+t}{2} a+\frac{1-t}{2} b\right)\right|^{q} d t \\
& \leqslant\|w\|_{[a, b], \infty} \frac{1}{3}\left(\left|f^{\prime}(a)\right|^{q} \int_{0}^{1} h\left(\frac{1+t}{2}\right) d t+\left|f^{\prime}(b)\right|^{q} \int_{0}^{1} h\left(\frac{1-t}{2}\right) d t\right) .
\end{aligned}
$$

Using the last two inequalities we obtain

$$
\left|\frac{1}{6(b-a)}\left[f(a)+f(b)+4 f\left(\frac{a+b}{2}\right)\right] \int_{a}^{b} w(x) d x-\frac{1}{b-a} \int_{a}^{b} w(x) f(x) d x\right|
$$




$$
\begin{aligned}
\leqslant & \frac{b-a}{2}\|w\|_{[a, b], \infty} \cdot\left(\frac{5}{36}\right)^{1-\frac{1}{q}} \cdot\left(\frac{2}{3}\right)^{\frac{1}{q}} \cdot\left\{\left(\left|f^{\prime}(a)\right|^{q} \int_{0}^{\frac{1}{2}} h(t) d t+\left|f^{\prime}(b)\right|^{q} \int_{\frac{1}{2}}^{1} h(t) d t\right)^{\frac{1}{q}}\right. \\
& \left.+\left(\left|f^{\prime}(a)\right|^{q} \int_{\frac{1}{2}}^{1} h(t) d t+\left|f^{\prime}(b)\right|^{q} \int_{0}^{\frac{1}{2}} h(t) d t\right)^{\frac{1}{q}}\right\} .
\end{aligned}
$$

This completes the proof.

Corollary 2.12. In Theorem 2.11, if we take $\mathrm{h}(\mathrm{t})=\mathrm{t}$, then inequality (2.3) becomes the following inequality for convex functions:

$$
\begin{aligned}
& \left|\frac{1}{6(b-a)}\left[f(a)+f(b)+4 f\left(\frac{a+b}{2}\right)\right] \int_{a}^{b} w(x) d x-\frac{1}{b-a} \int_{a}^{b} w(x) f(x) d x\right| \\
& \leqslant \frac{b-a}{8}\|w\|_{[a, b], \infty} \cdot\left(\frac{5}{9}\right)^{1-\frac{1}{q}} \cdot\left(\frac{1}{3}\right)^{\frac{1}{q}} \cdot\left\{\left(\left|f^{\prime}(a)\right|^{q}+3\left|f^{\prime}(b)\right|^{q}\right)^{\frac{1}{q}}+\left(3\left|f^{\prime}(a)\right|^{q}+\left|f^{\prime}(b)\right|^{q}\right)^{\frac{1}{q}}\right\} .
\end{aligned}
$$

Corollary 2.13. Suppose $\mathrm{h}(\mathrm{t})=\mathrm{t}^{\mathrm{s}}, \mathrm{s} \in(0,1]$ in Theorem 2.11, we have the following inequality for s-convex functions:

$$
\begin{aligned}
& \left|\frac{1}{6(b-a)}\left[f(a)+f(b)+4 f\left(\frac{a+b}{2}\right)\right] \int_{a}^{b} w(x) d x-\frac{1}{b-a} \int_{a}^{b} w(x) f(x) d x\right| \\
& \leqslant \frac{b-a}{2}\|w\|_{[a, b], \infty} \cdot\left(\frac{2}{(s+1) 3}\right)^{\frac{1}{q}}\left(\frac{5}{36}\right)^{1-\frac{1}{q}}\left\{\left(\left(\frac{1}{2}\right)^{s+1}\left|f^{\prime}(a)\right|^{q}+\left(1-\left(\frac{1}{2}\right)^{s+1}\right)\left|f^{\prime}(b)\right|^{q}\right)^{\frac{1}{q}}\right. \\
& \left.\quad+\left(\left(1-\left(\frac{1}{2}\right)^{s+1}\right)\left|f^{\prime}(a)\right|^{q}+\left(\frac{1}{2}\right)^{s+1}\left|f^{\prime}(b)\right|^{q}\right)^{\frac{1}{q}}\right\} .
\end{aligned}
$$

Theorem 2.14. Let $f:[a, b] \rightarrow R$ be a differentiable mapping on $(a, b)$ and $f^{\prime} \in L([a, b])$ with $a<b$ and $w:[a, b] \rightarrow R$ be continuous and symmetric to $\frac{\mathrm{a}+\mathrm{b}}{2}$. If $\left|\mathrm{f}^{\prime}\right|^{\mathrm{q}}$ is $\mathrm{h}$-convex on $[\mathrm{a}, \mathrm{b}]$ and $\mathrm{q}>1$, then the following inequality holds:

$$
\begin{aligned}
& \left|\frac{1}{6(b-a)}\left[f(a)+f(b)+4 f\left(\frac{a+b}{2}\right)\right] \int_{a}^{b} w(x) d x-\frac{1}{b-a} \int_{a}^{b} w(x) f(x) d x\right| \\
& \leqslant \frac{b-a}{12}\|w\|_{[a, b], \infty} \cdot\left(\frac{1+2^{p+1}}{(p+1) 3}\right)^{\frac{1}{p}}\left(\int_{0}^{1} h(t) d t\right)^{\frac{1}{q}}\left\{\left(\left|f^{\prime}\left(\frac{a+b}{2}\right)\right| q+\left|f^{\prime}(b)\right|^{q}\right)^{\frac{1}{q}}\right. \\
& \left.\quad+\left(\left|f^{\prime}(a)\right|^{q}+\left|f^{\prime}\left(\frac{a+b}{2}\right)\right|^{q}\right)^{\frac{1}{q}}\right\},
\end{aligned}
$$

where $\frac{1}{\mathrm{p}}+\frac{1}{\mathrm{q}}=1$. 
Proof. From Lemma 2.1 and using the Hölder's integral inequality, we have

$$
\begin{aligned}
& \left|\frac{1}{6(b-a)}\left[f(a)+f(b)+4 f\left(\frac{a+b}{2}\right)\right] \int_{a}^{b} w(x) d x-\frac{1}{b-a} \int_{a}^{b} w(x) f(x) d x\right| \\
& \leqslant \\
& \quad \frac{b-a}{2}\left\{\left(\int_{0}^{1}\left|\frac{1}{2} \int_{0}^{t} w\left(\frac{1-s}{2} a+\frac{1+s}{2} b\right) d s-\frac{1}{3} \int_{0}^{1} w\left(\frac{1-s}{2} a+\frac{1+s}{2} b\right) d s\right|^{p} d t\right)^{\frac{1}{p}}\right. \\
& \quad \times\left(\int_{0}^{1}\left|f^{\prime}\left(\frac{1-t}{2} a+\frac{1+t}{2} b\right)\right| q d t\right)^{\frac{1}{q}} \\
& +\left(\int_{0}^{1}\left|\frac{1}{3} \int_{0}^{1} w\left(\frac{1+s}{2} a+\frac{1-s}{2} b\right) d s-\frac{1}{2} \int_{0}^{1} w\left(\frac{1+s}{2} a+\frac{1-s}{2} b\right) d s\right|^{p} d t\right)^{\frac{1}{p}} \\
& \left.\quad \times\left(\int_{0}^{1}\left|f^{\prime}\left(\frac{1+t}{2} a+\frac{1-t}{2} b\right)\right|^{q} d t\right)^{\frac{1}{q}}\right\}
\end{aligned}
$$

Since $\left|f^{\prime}\right|^{q}$ is h-convex, by (1.3) we have

$$
\int_{0}^{1}\left|f^{\prime}\left(\frac{1-t}{2} a+\frac{1+t}{2} b\right)\right|^{q} d t=\frac{2}{b-a} \int_{\frac{a+b}{2}}^{b}\left|f^{\prime}(x)\right|^{q} d x \leqslant\left[\left|f^{\prime}\left(\frac{a+b}{2}\right)\right|^{q}+\left|f^{\prime}(b)\right|^{q}\right] \int_{0}^{1} h(t) d t,
$$

and

$$
\int_{0}^{1}\left|f^{\prime}\left(\frac{1+t}{2} a+\frac{1-t}{2} b\right)\right|^{q} d t=\frac{2}{b-a} \int_{a}^{\frac{a+b}{2}}\left|f^{\prime}(x)\right|^{q} d x \leqslant\left[\left|f^{\prime}(a)\right|^{q}+\left|f^{\prime}\left(\frac{a+b}{2}\right)\right|^{q}\right] \int_{0}^{1} h(t) d t .
$$

Therefore we obtain

$$
\begin{aligned}
& \left|\frac{1}{6(b-a)}\left[f(a)+f(b)+4 f\left(\frac{a+b}{2}\right)\right] \int_{a}^{b} w(x) d x-\frac{1}{b-a} \int_{a}^{b} w(x) f(x) d x\right| \\
& \leqslant \frac{b-a}{12}\|w\|_{[a, b], \infty} \cdot\left(\frac{1+2^{p+1}}{(p+1) 3}\right)^{\frac{1}{p}} \cdot\left(\int_{0}^{1} h(t) d t\right)^{\frac{1}{q}} \cdot\left\{\left(\left|f^{\prime}\left(\frac{a+b}{2}\right)\right|^{q}+\left|f^{\prime}(b)\right|^{q}\right)^{\frac{1}{q}}\right. \\
& \left.\quad+\left(\left|f^{\prime}(a)\right|^{q}+\left|f^{\prime}\left(\frac{a+b}{2}\right)\right|^{q}\right)^{\frac{1}{q}}\right\}
\end{aligned}
$$

where $\frac{1}{p}+\frac{1}{q}=1$. We also note that

$$
\int_{0}^{1}\left|\frac{1}{2} t-\frac{1}{3}\right|^{p} d t=\int_{0}^{1}\left|\frac{1}{3}-\frac{1}{2} t\right|^{p} d t=\frac{2+2^{p+2}}{(p+1) \cdot 6^{p+1}} \frac{1}{p}
$$

This completes the proof. 
Remark 2.15. If we choose $h(t)=t$ or $h(t)=t^{s}, s \in(0,1]$ in Theorem 2.14, we obtain the inequalities for convex or s-convex functions respectively.

Remark 2.16. If we choose $h(t)=t, s \in(0,1]$ and $w(x)=1$ for each $x \in[a, b]$, then we obtain inequality (1.2) of Theorem 1.3.

\section{References}

[1] M. Alomari, M. Darus, S. S. Dragomir, New inequalities of Simpson's type for s-convex functions with applications, Res. Rep. Coll., 12 (2009), 18 pages. 1

[2] M. Alomari, S. Hussain, Two inequalities of Simpson type for quasi-convex functions and applications, Appl. Math. E-Notes, 11 (2011), 110-117.

[3] S. S. Dragomir, R. P. Agarwal, P. Cerone, On Simpson's inequality and applications, J. Inequal. Appl., 5 (2000), 533-579. 1

[4] Z. Liu, An inequality of Simpson type, Proc. R. Soc. Lond. Ser. A Math. Phys. Eng. Sci., 461 (2005), $2155-2158$.

[5] M. Matłoka, Some inequalities of Simpson type for h-convex functions via fractional integrals, Abstr. Appl. Anal., 2015 (2015), 5 pages.

[6] J. Pečarić, S. Varošanec, A note on Simpson's inequality for functions of bounded variation, Tamkang J. Math., 31 (2000), 239-242.

[7] J. Pečarić, S. Varošanec, A note on Simpson's inequality for Lipschitzian functions, Soochow J. Math., 27 (2001), 53-57. 1

[8] M. Z. Sarikaya, A. Saglam, H. Yildirim, On some Hadamard-type inequalities for h-convex functions, J. Math. Inequal., 2 (2008), 335-341. 1

[9] M. Z. Sarikaya, E. Set, E. Özdemir, On new inequalities of Simpson's type for convex functions, Res. Rep. Coll., 13 (2010), 13 pages. 1,1

[10] M. Z. Sarikaya, E. Set, E. Özdemir, On new inequalities of Simpson's type for s-convex functions, Comput. Math. Appl., 60 (2010), 2191-2199. 1, 1, 2.10

[11] E. Set, E. Özdemir, M. Z. Sarikaya, On new inequalities of Simpson's type for quasi-convex functions with applications, Tamkang J. Math., 42 (2012), 357-364. 1

[12] K.-L. Tseng, G.-S. Yang, S. S. Dragomir, On weighted Simpson type inequalities and applications, J. Math. Inequal., 1 (2007), 13-22. 1

[13] S. Varošanec, On h-convexity, J. Math. Anal. Appl., 326 (2007), 303-311. 1 